\title{
An Optimization-based Tool to Support the cost- effective Production of Software Architecture Documentation
}

\author{
Matias Nicoletti ${ }^{1}$, Silvia Schiaffino ${ }^{1}$, and J. Andrés Diaz Pace ${ }^{1}$ \\ ${ }^{1}$ ISISTAN (CONICET - UNCPBA), Campus Universitario, Paraje Arroyo Seco, \\ Tandil, 7000, Argentina \\ \{matias.nicoletti, silvia.schiaffino, \\ andres.diazpace\} @isistan.unicen.edu.ar
}

\begin{abstract}
Some of the challenges faced by most software projects are tight budget constraints and schedules, which often make managers and developers prioritize the delivery of a functional product over other engineering activities, such as software documentation. In particular, having little or low-quality documentation of the software architecture of a system can have negative consequences for the project, as the architecture is the main container of the key design decisions to fulfill the stakeholders' goals. To further complicate this situation, generating and maintaining architectural documentation is a non-trivial and time-consuming activity. In this context, we present a tool approach that aims at (i) assisting the documentation writer in their tasks and (ii) ensuring a cost-effective documentation process by means of optimization techniques. Our tool, called SADHelper, follows the principle of producing reader-oriented documentation, in order to focus the available, and often limited, resources on generating just enough documentation that satisfies the stakeholders' concerns. The approach was evaluated in two experiments with users of software architecture documents, with encouraging results. These results show evidence that our tool can be useful to reduce the documentation costs and even improve the documentation quality, as perceived by their stakeholders.
\end{abstract}

Keywords. software architecture; architecture documentation; multi-objective optimization; stakeholders; tool support; wikis.

Citation. Nicoletti, M., Schiaffino, S., and Diaz-Pace, J. A. (2015) An optimization-based tool to support the cost-effective production of software architecture documentation. Journal of Software: Evolution and Process (Wiley), 27: 674-699. doi: 10.1002/smr.1734 